\section{$\underset{\substack{\text { hommes } \\ \text { \& migrations }}}{ }$}

\section{Hommes \& migrations}

Revue française de référence sur les dynamiques

migratoires

1308 | 2014

Les Paris des migrants

\title{
Les Wenzhous de Paris et d'ailleurs
}

\section{Richard Beraha}

\section{OpenEdition}

Journals

\section{Édition électronique}

URL : http://journals.openedition.org/hommesmigrations/2994

DOI : 10.4000/hommesmigrations.2994

ISSN : 2262-3353

\section{Éditeur}

Musée national de l'histoire de l'immigration

\section{Édition imprimée}

Date de publication : 1 octobre 2014

Pagination : $55-63$

ISBN : 978-2-919040-29-2

ISSN : $1142-852 X$

\section{Référence électronique}

Richard Beraha, «Les Wenzhous de Paris et d'ailleurs », Hommes \& migrations [En ligne], 1308| 2014, mis en ligne le 01 octobre 2017, consulté le 01 mai 2019. URL : http://journals.openedition.org/ hommesmigrations/2994 ; DOI : 10.4000/hommesmigrations.2994 


\title{
LES WENZHOUS DE PARIS ET D'AILLEURS
}

par RICHARD BERAHA, ex-président de l'ex-association Hui Ji, consultant, enseignant à l'IEP (Paris).

\author{
Migration laborieuse, migration silencieuse. Les migrants \\ chinois originaires du district de Wenzhou demeurent discrets \\ dans la société française. Or la France constitue, depuis une \\ trentaine d'années, une destination privilégiée des réseaux \\ migratoires et commerciaux qui contribuent à la vitalité \\ industrielle du sud-est de la Chine. Entre Paris et cette région \\ chinoise, ces "paysans prolétaires commerçants" ont su \\ développer les conditions d'une ascension sociale, tout en \\ apportant leur contribution humaine et économique à la France, \\ sans jamais rompre les liens avec leur terre d'origine.
}

Environ 250000 Chinois dorigine du Zhejiang, plus exactement du district de Wenzhou, vivent en France, dont les deux tiers en région parisienne ${ }^{1}$. Ils représentent à peu près $70 \%$ des personnes originaires de République populaire de Chine et la moitié de ceux d'origine chinoise (les autres provenant du Viêtnam, du Laos et du Cambodge). Cette population reste cependant méconnue, cernée d'un halo de mystère et de préjugés flatteurs ou discriminants. Quelle est l'histoire singulière de l'intégration des Wenzhous danslasociétéfrançaise?Commentexpliquer leur économie transnationale et ses mutations récentes ? Quelles perspectives pour ces migrants et leurs enfants entre l'intégration dans une Répu- blique française de plus en plus craintive et l'attrait de leur pays d'origine en plein essor?

Telles sont les questions auxquelles nous allons tenter de répondre pour rendre compte de ce mouvement migratoire et social, tout en tentant de dissiper clichés et malentendus. Les résultats présentés ici ont pris forme dans le cadre d'une rechercheaction participative en France, en Chine et en Italie (région Île-de-France, PICRI²) que j'ai coordonnée, impliquant l'association Hui Ji, le Cadis (EHESS/ CNRS), ainsi que l'École normale du Zhejiang. La méthodologie, inspiréedes travauxdeWilliamFoote Whyte $^{3}$, consiste à impliquer des migrants à toutes les étapes du processus, de l'élaboration initiale à 
l'application pratique des résultats. La réciprocité interpersonnelle lie les informateurs principaux et les chercheurs autour d'objectifs de retombées pour l'action (médiation avec les pouvoirs publics, régularisations, actions d'intégration, actions envers les enfants, développement des commerces, recherches, etc. $)^{4}$.

\section{De la clandestinité \\ au commerce, sur les pas de leurs ancêtres}

Les premiers Wenzhous sont arrivés en France à la fin du XIXe siècle, rejoints en 1917 par quelques milliers de coolies, et entre les deux guerres par des marchands ambulants ${ }^{5}$. Cette petite communauté vivait dans l'îlot Chalon près de la gare de Lyon et a ensuite migré dans le III arrondissement (Arts-et-Métiers). Elle s'enrichit de l'apport de milliers de paysans prolétaires et commerçants dès le début des années 1980 avec des implantations sur les territoires de Belleville, du Nord-Est parisien et de Seine-Saint-Denis ${ }^{6}$. Ces

Les migrants arrivés Wenzhous ont accompadans les années 1980-2000

ont presque tous pu accéder, malgré leur faible

niveau d'éducation, à la classe moyenne grâce à l'entreprenariat. gné les flux marchands de la Chine vers l'Europe dès son entrée dans l'Organisation mondiale du commerce $(\mathrm{OMC})$, notamment dans le domaine du textile. Mais, à l'inverse de l'autre grande migration asiatique en France - Viêtnam, Laos et Cambodge -, en majorité de culture chinoise (du Guangdong) et installée dans le quartier chinois du XIII arrondissement et à Marne-la-Vallée, qui fuyait, elle, des violences politiques (boat people), ces Wenzhous n'ont été ni accueillis, ni intégrés. Arrivés pour une grande part sans visa - impossible à obtenir, donc sans papiers -, ils ont dû se cacher pendant plusieurs années de la police et des voleurs, travailler dans l'ombre de leur réseau social pour rembourser leur dette de passage, et vivre chichement, mais vivre cependant, en se mariant et en élevant leurs enfants, en économisant pour l'avenir. Quasiment en dehors de la société française s'est ainsi développée une culture de la clandestinité, qui leur est souvent reprochée, mais qui a été en fait fortement conditionnée par une clandestinité subie. Dans d'autres pays, alors que sont observées les mêmes formes de société endogène par réseau, les liens sociaux et économiques avec le pays d'accueil peuvent être beaucoup plus développés. En Italie, par exemple, où les régularisations ont été plus aisées et rapides, ces migrants contribuent à l'équilibre de la balance extérieure transalpine en exportant des produits italiens. Un phénomène qui reste marginal en France.

Aujourd'hui en grande majorité régularisés - il en resterait tout de même encore quelques dizaines de milliers privés de titre de séjour -, ces Chinois de Wenzhou occupent des niches économiques dans la restauration chinoise et japonaise, la maroquinerie, le textile, la bijouterie, et plus globalement dans l'industrie légère, le bazar, l'informatique et, depuis peu, les bars, les brasseries, les bars-tabacs et commerces alimentaires. Très entreprenants, les Wenzhous ont créé des centres de commerce de gros internationaux à Aubervilliers (où on compte environ un millier d'entreprises), dans le $\mathrm{XI}^{\mathrm{e}}$ arrondissement (autour de 600 entreprises, en particulier autour des rues Sedaine et Popincourt), dans le Marais et le Sentier (500 entreprises), environ 40000 commerces de détail répartis sur l'ensemble du territoire français, outre-mer compris. Scolarisés en France, leurs enfants peuvent intégrer par la suite des entreprises françaises ou reprendre les affaires des parents. Certains deviennent avocats, comptables, informaticiens, prestataires de service, dirigeants de sociétés d'intermédiation entre la France et la Chine. On constate également de plus en plus de "retours", migrations permanentes ou temporaires vers la Chine. 


\section{De Paris à toute l'Europe}

Les migrants arrivés dans les années 1980-2000 ont presque tous pu accéder, malgré leur faible niveau d'éducation, à la classe moyenne grâce à l'entreprenariat. Ils témoignent cependant d'un parcours d'intégration encore inabouti, ne mâ̂trisant généralement que très imparfaitement le français (pas plus que le mandarin pour certains, car ils s'expriment en dialecte). Ceux arrivés plus récemment - mais le flux migratoire de Wenzhous vers la France s'est aujourd'hui pratiquement tari -, souvent mieux formés, rencontrent pourtant plus de difficultés car ils doivent affronter des marchés du travail saturés, le ralentissement de l'économie française et le renchérissement des produits chinois, sans parler du repli identitaire hexagonal. Les échecs, vécus comme une humiliation collective par la famille, ne sont plus isolés et entraînent des situations individuelles quelquefois dramatiques sur le plan matériel, mais plus encore sur le plan psychologique. Ces mêmes familles, souvent depuis la France, se sont activées sur les marchés italien et espagnol, en Grèce, au Portugal, dans les pays d'Europe centrale et de l'Est. Il existe des communautés de commerçants de cette région dans tous les pays de la planète (ils exportent dans 204 pays !). Sur 7,5 millions d'habitants du district de Wenzhou, 2 millions se seraient dispersés en trente ans dans toutes les régions et grandes villes de Chine, en Europe, mais aussi et de plus en plus en Amérique du Sud et du Nord, en Afrique et dans tous les pays asiatiques.

À cette immense dispersion correspond une source très réduite car, dans les faits, les départs ne s'effectuent que depuis quelques "qiaoxiang", des villages montagneux aux habitus de migration ${ }^{7}$, dont parfois l'ensemble de la population active a migré. Par ailleurs, $95 \%$ de l'économie wenzhou est assurée par des entreprises privées ${ }^{8}$. Les exportations s'ef-

fectuent généralement entre compatriotes, dans des réseaux qui concurrencent aujourd'hui les grandes entreprises occidentales. Mais ces réseaux ont eu au moins deux mérites, d'où leur relatif succès. En premier lieu, des prix plus bas pour le consommateur (en revanche, peu de valeur ajoutée immatérielle, pas de marque). Ensuite, une forme de capitalisme accompagnée d'une large redistribution : dans une multinationale classique, le bénéfice revient à une poignée d'actionnaires, alors qu'ici il est partagé entre des milliers d'entreprises familiales de production, d'import-export, de gros et de détail, toutes en interaction dans des réseaux informels non limités, mais globaux et formant filière. Sur le terrain parisien, quand on observe les migrants à leur arrivée, honteux, laborieux, souvent exploités, assujettis à une dette, vivant dans des conditions d'hygiène précaires, s'entassant dans des logements exigus, enfermés parfois dans des centres de

Sur 7,5 millions d'habitants du district de Wenzhou, 2 millions se seraient dispersés en trente ans dans toutes les régions et grandes villes de Chine, en Europe, mais aussi et de plus en plus en Amérique du Sud et du Nord, en Afrique et dans tous les pays asiatiques. rétention, les images d'Épinal du misérabilisme ne durent qu'un temps, aussitôt contredites : comment expliquer que presque tous, en quelques années seulement, parviennent non pas à devenir milliardaires, mais à accéder à un enviable statut de classe moyenne, qui leur permet de prouver leur fiabilité économique et de faire suivre des études à leur progéniture ? Un saisissant paradoxe que nous proposons d'éclaircir.

\section{Trois facteurs de transformation}

D'importants travaux de recherche (chinois, américains ou européens) ont explicité ce processus de migration en chaîne, à la fois humaine, commerciale et capitalistique par la constitution de diasporas 
Chaque année, lors du Nouvel An chinois, ce bouddha protecteur de la famille et des commerçants, garant de la prospérité et de la réussite, est sorti du temple dénommé Autel du culte de Bouddha afin de participer au défilé et de gratifier le quartier de son aura bénéfique. (c) Gilles Crampes

commerçantes - chinoise, indienne, européenne et leur intégration paradoxale dans les sociétés d'accueili9. Cependant, pour saisir la particularité de la migration wenzhou, il nous faut élargir notre vision dans l'espace et le temps et linsérer dans la période de globalisation contemporaine. Trois phénomènes émergents sont significatifs de cette nouvelle réalité et donnent sens aux formes singulières de migration et d'intégration des Wenzhous en France en ce début du XXI siècle. Trois processus de trans-

formation - souvent silencieux ${ }^{10}$ - sont à l'œuvre : historique, social et individuel.

Tout d'abord, un processus historique, avec l'essor de la Chine. Depuis 2008, ce pays affiche sa puissance sur la scène mondiale. Les Jeux olympiques, l'Exposition universelle de Shanghai, les investissements en Europe, l'attractivité de l'immense marché chinois pour les entreprises du reste du monde, les nouveaux médias chinois et leur mâ̂trise de l'Internet ont modifié les représentations et la façon dont les journaux s'expriment sur ce pays et aussi sur la diaspora chinoise française (les deux apparaissant souvent liés sans discernement). 
Le deuxième processus est social : la présence dans les rues de nombreuses villes françaises de milliers de commerces chinois de détail, de gros, de restaurants, de services, ainsi que celle des enfants de migrants dans les écoles. Cette visibilité permet de multiplier les interactions avec cette nouvelle migration, témoin d'une globalisation par le bas. Notons également la présence de 60000 étudiants chinois originaires de toutes les régions de Chine, qui se mélangent - encore peu - à la diaspora.

En troisième lieu, nous observons une transformation individuelle : l'expression d'une volonté individuelle affichée, quoique discrètement, tant par les adultes que par leurs enfants, de sortir de l'ombre, de devenir sujets et acteurs, en participant à des luttes, en s'associant au débat public et politique, en revendiquant leur appartenance à la république comme leur rôle de pont entre deux sociétés et deux cultures.

\section{Une mondialisation par le bas}

Les trois derniers siècles ont été marqués pour les Chinois par de nombreuses vicissitudes. Le régime maoïste, qui apportait enfin l'indépendance au pays, a suscité violences, disettes, confiscations, incertitudes, méfiances, peurs. Il a notamment tenté - en vain - d'anéantir les traditions (les quatre "vieilleries" : idées, culture, coutumes, habitudes) et le marché économique privé, ainsi que les religions coutumières ou chrétiennes dans une région appelée par certains la "Jérusalem de Chine". Ces souffrances séculaires ont été endurées dans les corps et les esprits et transmises - au delà des non-dits - de génération en génération.

Dès que Deng Xiaoping a lancé le slogan "Enrichissez-vous!", le fait d'avoir de la famille à l'étranger a été perçu comme une chance (alors qu'il s'agissait avant d'une trahison). Dès l'ouverture des marchés, avec des passeports faciles à acquérir, ces Wenzhous des qiaoxiang ont sauté le pas. Ils ont, en fait, reproduit un habitus ancestral, car dans la Chine ancienne, toutes les maisons (appelées aussi "clans", large parentèle familiale avec un même patronyme) avaient trois activités : la terre, l'artisanat et le commerce, le plus souvent ambulant pour aller vendre produits de la ferme et fabrications artisanales.

Ces Wenzhous vivaient dans des régions montagneuses et isolées, organisés en clans hiérarchisés et solidaires. Ils survivaient aux troubles sociaux et politiques, souvent en conflit larvé avec le pouvoir central, soudés par le culte des ancêtres, les divinités locales (20\% sont chrétiens aujourd'hui, principalement protestants évangélistes), les rituels familiaux, un système holiste

de don et de contre-don, une

Contrairement à ce qui morale d'honneur, une tradis'écrit parfois, ces migrants tion de forte mobilité ${ }^{11}$.

Ils furent donc les premiers à se lancer dans le commerce, dès que cela a été possible en Chine. ne sont en rien liés à des mafias internationales, qui séjournent dans d'autres régions que Ils ont créé le premier centre le Zhejiang. commercial à Pékin, le Village Zhejiang ${ }^{12}$, engendrant rapidement, en dehors de toute assistance publique, cliniques, transports, banques, investissements immobiliers...

Le Parti communiste a même conduit une étude visant à démontrer que le capitalisme familial et la liberté d'entreprendre dans ces régions portuaires, montagneuses et industrieuses, en lien aveclétranger depuis toujours, sont favorables au développement des zones rurales ${ }^{13}$. Cette région si dynamique, essentiellement dominée par des réseaux locaux, se situe parmi les premières en terme de niveau de vie, avec une croissance continue 
supérieure à deux chiffres depuis trente ans et ce, grâce en grande partie aux exportations facilitées par les migrants éparpillés et à une organisation de PME en réseau, chaque village ayant ses spécialités, chaque métier ses filières et ses propres associations.

Au cours des années de forte émigration (19802000), être citoyen d'un village de montagne et ne pas disposer de "hukou" urbain (permis de citoyen donnant droit à des avantages sociaux) rendait quasi impossible l'accès à l'université. Les jeunes, filles et garçons, entre 16 et 25 ans, ont alors capté l'appel de leurs parents d'outre-mer, déjà installés et commerçants. Ces derniers avaient besoin de main-d'œuvre en France et aussi en Chine pour développer leurs affaires. Certains villages se sont alors littéralement vidés. Contre 10000 ou 15000 euros, des passeurs, "têtes de serpent", proposaient aux candidats le grand voyage. Ce ne sont donc pas les plus pauvres qui sont partis, ni les plus riches, mais ceux dont la famille avait tout de même quelques ressources pour investir dans un changement de vie. Contrairement à ce qui s'écrit parfois, ces migrants ne sont en rien liés à des mafias internationales, qui

Dès la régularisation acquise, en général après

une dizaine d'années de rétrogradation sociale, le salarié cherche à devenir patron. séjournent dans d'autres régions que le Zhejiang.

La somme due au passeur est réglée en deux fois par la famille du migrant, la moitié au départ, le reste à l'arrivée. Ensuite, le primo-arrivant travaille dur pendant deux ou trois années dans son réseau pour rembourser sa dette (cuisinier, ouvrier textile...), se marie généralement avec une compatriote d'un village voisin présentée par les parents, tisse et soude autour de sa jeune famille un nouveau groupe social, dans lequel il trouvera entraide, solidarité, attaches affectives, sécurité et confiance. Ses enfants, 2 en moyenne, constituent souvent le seul lien avec la société française grâce à l'école. Les parents ne font que très peu appel à l'aide sociale, mais consacrent une part significative de leurs revenus à des dons rituels (mariage, naissance, ouverture d'une boutique), sommes que le futur entrepreneur retrouvera peu ou prou lorsqu'il en aura le plus besoin. Il s'agit en quelque sorte d'une forme d'impôt informel favorisant la fluidité du capital et donc le développement économique par la confiance entre les acteurs. Dès la régularisation acquise, en général après une dizaine d'années de rétrogradation sociale, le salarié cherche à devenir patron. Une autre étape s'ouvre à lui : être enfin reconnu comme citoyen et pouvoir à nouveau retourner au pays et renouer les liens familiaux distendus par la séparation. Patrons, en fait ils n'auront jamais cessé de l'être, car la plupart des sans-papiers travaillent à la tâche et de plus en plus à leur domicile, les ateliers clandestins, florissants dans les années 1990 et 2000, ayant aujourd'hui pratiquement disparu.

\section{Un mimétisme migratoire, entre ascension et déclin}

La mobilisation du capital économisé et des prêts, la plupart sans intérêt, octroyés par les proches, vont permettre de devenir, seul ou en association, mais presque toujours en couple, gérant d'un commerce alimentaire, d'une boutique de bazar ou de textile. Le nouvel entrepreneur copie le plus souvent sur son ex-employeur, issu de sa famille ou de ses proches ; il s'approvisionne dans des filières chinoises avec la possibilité d'obtenir un crédit fournisseur. Beaucoup d'entre eux en restent à ce stade, car leur niveau de connaissance de la société française demeure rudimentaire. Ce sont les enfants qui, par la suite, se chargeront de moderniser l'entreprise s'ils n'ont pas fui le milieu professionnel de leurs parents, ce qui est souvent une de leurs aspirations. Cependant, ils parviennent rarement à réaliser leur émancipation, faute d'avoir pu trouver plus attrayant ailleurs, c'est-à-dire sur le marché du travail français. Une minorité accède toutefois aux formes les plus développées du capitalisme chinois 


\section{Asiatiques à Paris et Petite couronne en 1999}
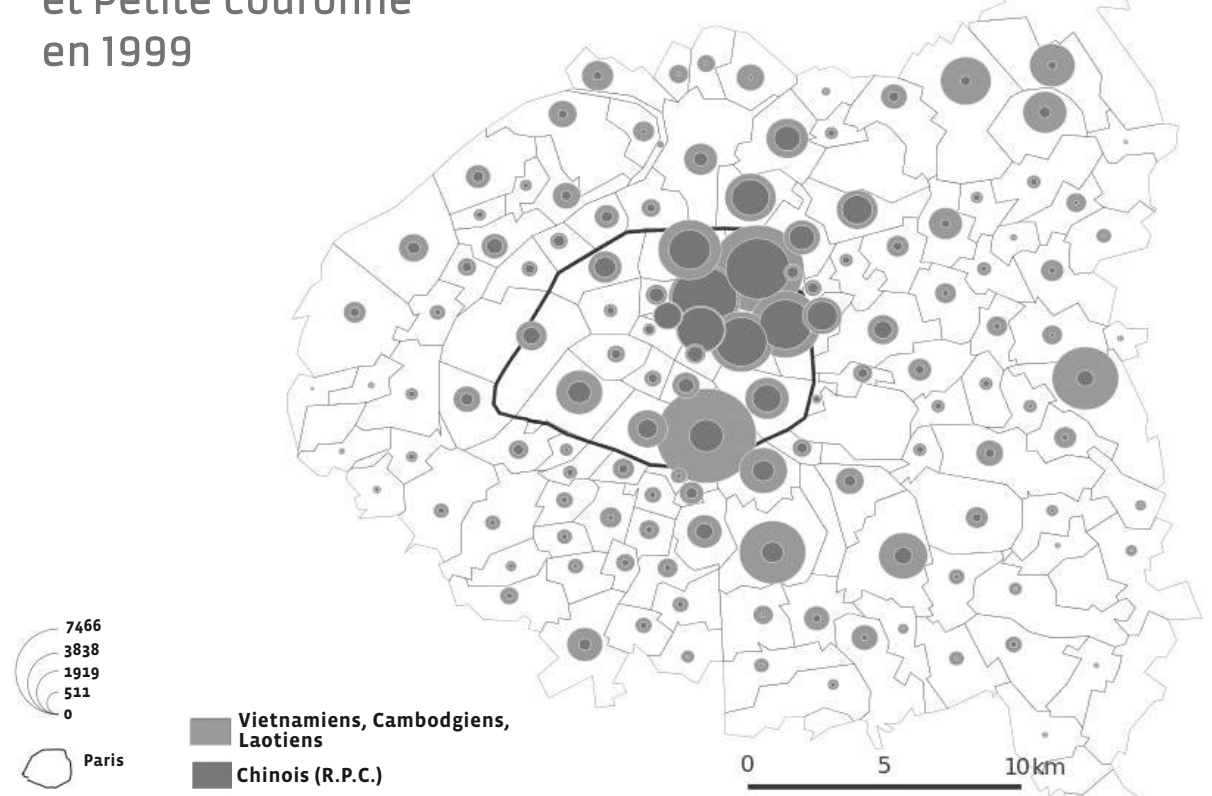

Vietnamiens, Cambodgiens,

Source : Insee, Recensement général de la population (RGP), 1999.

et international en créant des multinationales et en vivant entre les deux pays.

Ce système d'accession de prolétaires à la classe moyenne est rendu possible par une organisation sociale spécifique, en réseau, bâtie autour de la famille et entretissée de liens villageois, donc ancrée dans le local, mais un local qui en réalité devient vite global puisqu'il s'étend par ramifications à l'ensemble de la planète. Pourtant, ce système mimétique est aujourd'hui en déclin. En effet, il s'appuie sur des réseaux primaires (non forcément partagés par la seconde génération), sur une société régie par un code social coutumier (l'individualisme contemporain y voit un enfermement) et sur des pratiques organisationnelles et commerciales traditionnelles non adaptées au monde complexe, immatériel et technologique de ce nouveau siècle. De ce fait, les aînés parviennent difficilement à faire confiance à la jeune génération, à diversifier leur recrutement (embauche de non-Chinois, d'étudiants chinois diplômés). Ils rechignent aussi à créer une marque, une franchise, à se diversifier et à profiter de l'immense marché chinois en vendant des produits français à valeur ajoutée. Cette microéconomie de petits produits à petits prix, disponibles en grande variété et surtout en grande quantité, semble aujourd'hui en difficulté, concurrencée pour l'approvisionnement par des pays à plus bas prix (Bangladesh, Éthiopie, Cambodge) et sur les marchés par des acteurs globaux ou locaux plus 
imaginatifs et mieux organisés. D’ailleurs, la région vit depuis 2010 une succession de crises financières et de réorganisations de son tissu industriel, et elle est redevenue, comme au début des années 1980, une région d'expérimentation des réformes (notamment, en 2012, une réforme bancaire sur le financement des PME et les investissements privés à l'étranger).

\section{Paris, l'émergence de mobilisations sociales}

Les modes d'expression de ces Wenzhous de Paris et d'ailleurs sont marqués par un paradoxe. D'une part, l'identité chinoise est souvent mise en avant dans les commerces ; d'autre part, la discrétion reste une règle de conduite. Vertu confucéenne, c'est aussi un réflexe naturel pour tout Chinois devant l'inconnu de la société française et face aux incertitudes et aux craintes du monde chinois qui exerce un contrôle discret

Les modes d'expression de ces Wenzhous de Paris et d'ailleurs sont marqués par un paradoxe. D'une part, l'identité chinoise est souvent mise en avant dans les commerces; d'autre part, la discrétion reste une règle de conduite.

économique à Paris, chaque individu se situe à un stade particulier sur le plan social. Clandestin, précaire et exploité par des patrons chinois ou d'autres nationalités, sans citoyenneté, le primo-arrivant se mure dans le silence. Cependant, ses relations avec les clients ou l'administration tatillonne, l'apprentissage de la confrontation à l'Autre et la demande d'accès aux droits les plus élémentaires ont des chances de faire émerger, sous certaines conditions, notamment la maîtrise de la langue, le "sujet acteur". Cela fut le cas dès 1996 et jusqu'en 2000, à Paris, avec le $3^{\text {e }}$ collectif $^{14}$ et sa participation au mouvement des sans-papiers de Saint-Bernard. Puis, dès 2002 et jusqu'en 2010, avec le collectif Multitude qui s'est transformé en association Hui $\mathrm{Ji}^{15}$, œuvrant pour un mouvement social de médiation dans le quartier de Belleville. Créé par Liwen Dong, ce regroupement a permis la régularisation de centaines de familles, tout en leur apportant les services dont elles avaient besoin pour mieux s'insérer avec leurs enfants dans notre société. Hui Ji a enfin contribué à l'émergence des Wenzhous en les faisant mieux connaitre, en intervenant dans les médias et en accompagnant des regroupements associatifs, comme l'Association des jeunes Chinois de France ${ }^{16}$. En juin 2010, une manifestation contre l'insécurité ${ }^{17}$ regroupe 20000 Chinois à Belleville. Des voitures de police sont renversées et brûlées, engendrant stupeur et incompréhension. Dès 2008, Hui Ji avait pourtant publié une étude adressée à la Préfecture de police et à la Mairie, recensant les faits violents à Paris et en SeineSaint-Denis ciblant spécifiquement une population démunie, féminine, qui à l'époque ne pouvait porter plainte car elle était en situation irrégulière en France.

À partir de l'expérience de Hui Ji dans la médiation sociale intercommunautaire, une série de mesures ont été proposées pour mieux protéger ces migrants, notamment la création de postes de médiateur ainsi qu'un accueil spécifique en langue chinoise pour les victimes. Une revendication accordée un an plus tard suite à une seconde manifestation en juin 2011 sur le même thème, même si les formes organisationnelles avaient évolué, comme l'analyse avec finesse Ya-Han Chuang, doctorante chinoise à Paris. Celle-ci a aussi étudié le mouvement orchestré par la CGT ${ }^{18}$, qui a mobilisé des travailleurs clandestins chinois, faisant apparaitre les contradictions laborieusement surmontables entre la vision d'un salarié et celle d'un futur entrepreneur. 


\section{Une parole publique encore timide}

Ce qui caractérise tous ces mouvements et les réunit, c'est qu'ils ne s'inscrivent qu'avec difficulté dans le temps. Ce sont des soubresauts qui font bouger les lignes, concourent aux transformations silencieuses, mais n'aboutissent pas à des mouvements pérennes portés par des organisations représentatives. Car la société chinoise en France reste fragmentée en centaines de regroupements, le plus souvent autour du village d'origine, d'intérêts catégoriels (entre autres, association de commerçants), de classes d'âge et de genre, de buts culturels ou cultuels, et de plus en plus en lien avec le pays par l'ambassade et les étudiants chinois en France. Cette concurrence des intérêts, laminée par des horaires de travail importants pour tous, laisse peu de temps au bénévolat et à l'action désintéressée, et rend difficile l'expression collective, comme le démontrent les multiples associations dont certaines se veulent pourtant représentatives ${ }^{19}$. Créés à partir des années 2000 avec une inflation après les manifestations de Belleville, elles se caractérisent, en dehors de l'Association des jeunes Chinois de France (AJCF), par une absence de prise de parole publique. En revanche, notons l'élection de deux jeunes Chinois sur les listes de gauche de la Mairie de Paris : Olivier Wang dans le XIX', avocat et cofondateur de l'AJCF, et Weiming Martin Shi, commerçant et acteur social à Belleville dans le XXe. Ce n'est pas un hasard si l'un et l'autre ont été désignés comme délégués au commerce. Les liens entre l'UMP et certaines personnalités de la communauté ont été forts, mais cantonnés à une petite élite, sur- tout pendant la période sarkozyste, notamment après la signature d'un mémorandum entre ce parti et le Parti communiste chinois. Sont apparues ici les difficultés à faire cohabiter dans un même mouvement les Wenzhous et la seconde génération des migrants d'Asie du Sud-Est, impliquée depuis plus d'années en politique.

\section{Conclusion}

Dans l'évolution conduisant des migrants enfermés au départ dans leur groupe social comme dans ses hiérarchies (visibles et invisibles) jusqu'à l'autonomie et à l'adaptation à leur société d'accueil, les femmes jouent un rôle clé. L'un des bienfaits du communisme chinois a été de libérer la femme du patriarcat traditionnel, et nous constatons qu'elles sont en France plus rapidement intégrées sur le plan éducatif, économique et social que les hommes ${ }^{20}$. Dans ce long parcours d'acculturation et de socialisation, l'observation nous conduit aussi à percevoir la souffrance des individus dans leurs émotions et leur santé psychique, la difficulté des relations entre les générations, ce dont pâtissent le plus les personnes âgées ${ }^{21}$. Enfin, cette capacité de devenir acteur semble se déployer depuis 2012 grâce aux nouveaux réseaux sociaux chinois (We Chat, QQ), largement utilisés en Chine et au sein de la diaspora chinoise dans le monde (qui totalise environ 50 millions de personnes). Ils constituent une opportunité nouvelle, car sans "guanxi" (relations interpersonnelles entre deux individus symbolisant le networking), point de succès - ni vraiment de sujet potentiellement acteur - dans le monde chinois, en Chine comme en Occident. 